\title{
Down-regulation of CacyBP is associated with poor prognosis and the effects on COX-2 expression in breast cancer
}

\author{
FANG NIE, XIAO-LING YU, XIAO-GE WANG, YU-FU TANG, LING-LING WANG and LIE MA \\ Department of Respiratory Medicine, the Fourth Affiliated Hospital of China Medical University, \\ No. 4 Chongshandong Road, Yuhong District, Shenyang City, Liaoning Province 110032, P.R. China
}

Received May 19, 2010; Accepted July 16, 2010

DOI: 10.3892/ijo_00000777

\begin{abstract}
Calcyclin-binding protein (CacyBP) is a tumor suppressor in gastric and renal cell carcinoma, but an oncogene in pancreatic cancer. However, the function of CacyBP in breast cancer has not been well elucidated. In this study, we explored the clinical relevance of CacyBP and investigated the relationship between CacyBP and COX-2 in breast cancer. Immunohistochemical analysis in 172 cases of breast tissues showed that the positive rate of CacyBP protein expression in normal breast tissues (NBT) $(89.3 \%)$ was higher than that in invasive ductal carcinoma (IDC) $(56.1 \%)(\mathrm{P}<0.05)$. RT-PCR and Western blot analysis showed that CacyBP mRNA and protein expression were significantly lower in tumor tissues as compared to those in the corresponding non-tumorous tissues $(\mathrm{P}<0.05)$. The expression trend of $\mathrm{COX}-2$ was opposite with CacyBP in breast carcinogenesis. Moreover, the CacyBP expression was significantly negatively associated with the COX expression in the 132 breast cancer samples (correlation coefficient $=0.505, \mathrm{P}<0.001)$. The clinicopathological data analysis in 132 breast cancer samples showed that CacyBP expression was positively correlated with well differentiated samples $(\mathrm{P}=0.021)$, low pathologic TNM stage $(\mathrm{P}=0.009)$, and no lymphatic metastasis $(\mathrm{P}=0.027)$ of patients with breast cancer. Furthermore, reduced CacyBP expression was associated with poor prognosis. Knockdown of CacyBP gene using siRNA enhanced the proliferation and invasion ability of breast cancer cells, which was dependent on COX-2 expression. In conclusion, CacyBP regulation of COX-2 expression may play an important role in human breast carcinogenesis. Restoration of CacyBP gene is a potential therapeutic target of breast cancer.
\end{abstract}

Correspondence to: Dr Xiao-Ge Wang, Department of Respiratory Medicine, the Fourth Affiliated Hospital of China Medical University, No. 4 Chongshandong Road, Yuhong District, Shenyang City, Liaoning Province 110032, P.R. China

E-mail: cm4hwxgn2005@126.com

Key words: CacyBP, COX-2, breast cancer, prognosis, proliferation, invasion

\section{Introduction}

Breast cancer is among the leading causes of malignancyrelated death in the women all over the world. Although chemotherapy, radiation therapy and immunotherapy have been employed $(1,2)$, cancer cells remain resistant to these treatments and subsequently survive. It is urgent to look for useful biomarkers and identify novel therapeutic targets involved in the process of breast carcinogenesis.

CacyBP, which was used as a target of S100A family, was first identified in Ehrlich ascites tumor cells (3-5). CacyBP was expressed in a wide range of normal and tumor tissues (6). CacyBP was reported involved in inhibiting proliferation and invasion of gastric cancer cells through down-regulating ß-catenin expression (7). CacyBP antisense oligodeoxynucleotides also inhibited implantation in vivo and the apoptosis of endometrial stromal cells (8). CacyBP overexpression was observed in renal cell carcinoma and its expression associated with the suppression of cell growth and tumorigenesis (9). It was also reported that CacyBP expression was associated with aggressive phenotype of pancreatic cancer (10). These observations suggested that CacyBP might have important roles in tumorigenesis. However, the pathologic roles of CacyBP in breast cancer remain unclear and our study aimed to explore the effect of CacyBP in breast carcinogenesis and the related mechanism.

In the present study, we examined CacyBP expression in breast tissues and analyzed the relationship between CacyBP expression, COX-2 expression and clinicopathological factors of patients with breast cancer. We also explored the effects of CacyBP gene on COX-2 expression, cell proliferation and invasion in breast cancer cell lines using small interfering RNAs.

\section{Patients and methods}

Patients and specimens. Paraffin specimens $(\mathrm{n}=172)$ were obtained from patients with breast cancer who underwent curative surgical resection without having chemotherapy or radiation therapy in the Fourth Afiliated Hospital of China Medical University between 2004 and 2007. Follow-up data were obtained from review of the patients'medical record. Informed consent was obtained prior to surgery from all enrolled patients. According to World Health Organization 
breast carcinoma histological classification criteria (2003), there were 40 cases of normal breast tissues (NBT) and 132 cases of invasive ductal carcinoma (IDC). The TNM staging system of the International Union Against Cancer (UICC) in 2003 was used to classify specimens as stages I+II $(\mathrm{n}=78)$, and III+IV $(\mathrm{n}=54)$.

Thirty-eight cases (of the 132 cases) of tumor and paired non-tumorous tissues (distant from the primary tumor) of the same case were quickly frozen in $-70^{\circ} \mathrm{C}$ until protein and RNA extraction. Clinicopathological information of the patients on tumor size, histological type, differentiation, stage and lymph node metastasis was obtained from patient records, and summarized in Tables II and III.

Immunohistochemistry. The tissues were fixed with $10 \%$ neutral formalin, embedded in paraffin, and $4-\mu \mathrm{m}$-thick sections were prepared. Immunostaining was performed by the streptavidin-peroxidase (S-P) method (Ultrasensitive ${ }^{\mathrm{TM}}$, MaiXin, Fuzhou, China). The samples were incubated with CacyBP polyclonal antibody (1:100; Santa Cruz Biotechnology Inc., Santa Cruz, CA, USA) and COX-2 monoclonal antibody (1:100; Santa Cruz Biotechnology). For negative control, the primary antibodies were replaced by non-immune serum. Staining for both antibodies was performed at $4^{\circ} \mathrm{C}$ overnight. Biotinylated goat anti-mouse serum IgG was used as a secondary antibody. After washing, the sections were incubated with streptavidin-biotin conjugated with horseradish peroxidase, and the peroxidase reaction was developed with 3,3'-diaminobenzidine tetrahydrochloride. Counterstaining with hematoxylin was performed and the sections were dehydrated in ethanol before mounting.

Evaluation of CacyBP and COX-2 immunostaining. All of the stained sections were assessed by two independent pathologists who were unaware of the patients' clinical data. Five views were randomly examined per slide, and 100 cells were observed per view at magnification $\mathrm{x} 400$. The criteria of CacyBP immunohistochemical staining were according to Zhai et al (6). Cytoplasm/nuclear staining were considered positive, and it was scored on the following basis: 0 (no detectable staining $) ; 1+(<25 \%$ positive cells $) ; 2+(25-49 \%$ positive cells); $3+(50-74 \%$ positive cells $) ; 4+(>75 \%$ positive cells). When the sample was with scores $2-4$, we defined it as having the CacyBP high expression. When the sample was with scores $0-1$, we defined it as having the CacyBP low expression.

The criteria of COX-2 immunohistochemical staining were according to Ristimaki et al (11). The following scoring criteria of the tumor cells were agreed upon before the analysis: 0 , no staining; $1+$, weak diffuse cytoplasmic staining (may contain stronger intensity in $<10 \%$ of the cancer cells); $2+$, moderate to strong granular cytoplasmic staining in 10-90\% of the cancer cells; $3+,>90 \%$ of the tumor cells stained with strong intensity. When the sample was with scores $2-3$, we defined it as having the COX-2 high expression. When the sample was with scores $0-1$, we defined it as having the COX-2 low expression.

Cell culture. Human breast cancer cell lines MCF-7, MDAMB-231, and human breast epithelial MCF-10A cell line were maintained in Dulbcco's modifed Eagle's medium (DMEM)
(Gibco Inc., Grand Island, NY, USA) supplemented 10\% fetal bovine serum (Gibco) with $100 \mathrm{U} / \mathrm{ml}$ streptomycin and $100 \mathrm{U} / \mathrm{ml}$ penicillin in a humidified atmosphere with $5 \%$ of $\mathrm{CO}_{2}$. Cells were cultured to subconfluence until protein and RNA extraction.

RNA interference. CacyBP siRNA (h) (Cat SC-88504), COX-2 siRNA (h) (Cat SC-29279), and negative control siRNA (Cat SC-36869) were all from Santa Cruz Biotechnology. For transient transfection, cells were transfected with Lipofectamine 2000 according to the manufacturer's instructions (Invitrogen, Carlsbad, CA, USA).

Western blotting. Total protein from cells were extracted in lysis buffer and quantified using the Bradford method. Fifty micrograms of protein were separated by SDS-PAGE (12\%). After transferring to polyvinylidene fluoride (PVDF) membrane (Millipore, Billerica, MA, USA), the membranes were incubated overnight at $4^{\circ} \mathrm{C}$ with antibodies against CacyBP (1:1000), COX-2 (1:500) at $4^{\circ} \mathrm{C}$ overnight. After incubation with peroxidase-coupled anti-mouse IgG (Santa Cruz Biotechnology) at $37^{\circ} \mathrm{C}$ for $2 \mathrm{~h}$, bound proteins were visualized using ECL (Pierce Biotechnology, Rockford, IL, USA) and detected using BioImaging Systems (UVP Inc., Upland, CA, USA). The relative protein levels were calculated based on $\beta$-actin protein as a loading control. The experiments were repeated 3 times independently.

Reverse transcriptase-polymerase chain reaction (RT-PCR). Total RNA was extracted with TRIzol regent (Invitrogen). RT-PCR was performed with the AMV Ver3.0 kit (Takara, Shiga, Japan). The primer sequences and reaction conditions are shown in Table I. Thirty cycles were used for CacyBP, COX-2 and B-actin respectively. The PCR products were electrophoresed in a $2 \%$ agarose gel containing $0.1 \mu \mathrm{g} / \mu \mathrm{l}$ ethidium bromide. Then they were visualized and analyzed by the Bio-Imaging System (UVP). To provide a value for the transcriptional level of each gene, a grayscale intensity value was determined for each target band and normalized to $ß$-actin. Each experiment was repeated 3 times independently.

Matrigel invasion assay. Matrigel (BD Biosciences, MA, USA) and Transwell inserts of $8.0-\mu \mathrm{m}$ pore size (Corning Inc., NY, USA) were used for invasion assays. Briefly, Matrigel diluted with serum-free medium at a ratio of $1: 3$ $(100 \mu \mathrm{l})$ was added to the upper chamber, and $100 \mu \mathrm{l}$ of cell suspensions $\left(5 \times 10^{5}\right.$ cells $\left./ \mathrm{ml}\right)$ were added after the gel formed. Six hundred $\mu 1$ of medium containing $10 \%$ FBS was added to the lower chamber as the chemoattractant. After incubation for $48 \mathrm{~h}$, the filters were fixed with $100 \%$ methanol for $15 \mathrm{~min}$ and then subjected to hematoxylin staining. The cells that invaded and moved onto the lower surface of the filter membrane were counted in 10 random high power fields (x400) by an inverted microscope. The experiment was repeated 3 times and the data are shown as mean \pm standard deviation (SD).

MTT assay. Cells were plated in 96-well plates in the medium containing $10 \%$ FBS for $24 \mathrm{~h}$ after transfection. For quantitation of cell viability, cultures were stained after 4 days 
Table I. Primer sequences and reaction conditions of RT-PCR.

\begin{tabular}{llcr}
\hline Gene & \multicolumn{1}{c}{ Primer sequence $\left(5^{\prime}-3^{\prime}\right)$} & Length (bp) & Temperature $\left({ }^{\circ} \mathrm{C}\right)$ \\
\hline CacyBP & F: CTCCCATTACAACGGGCTAT & 359 & 55 \\
& R: TTCAGTGTCATAGGAGGG & & \\
COX-2 & F: TTCAAATGAGATTGTGGGAAAATTGCT & 299 \\
R: AGATCATCTCTGCCTGAGTATCTT & F: AGAGCTACGAGCTGCCTGAC & 308 \\
& R: AGTACTTGCGCTCAGGAGGA & & 54 \\
\hline
\end{tabular}

Table II. Relationships between CacyBP, COX-2 expression and clinicopathological facors in 132 cases of invasive ductal carcinoma.

\begin{tabular}{|c|c|c|c|c|c|c|c|c|c|}
\hline \multirow[b]{2}{*}{$\begin{array}{l}\text { Clinicopatho- } \\
\text { logical factors }\end{array}$} & \multirow[b]{2}{*}{$\mathrm{n}$} & \multicolumn{4}{|c|}{ СасуBP } & \multicolumn{4}{|c|}{$\mathrm{COX}-2$} \\
\hline & & + & $\begin{array}{l}\text { Positive } \\
\text { rate }(\%)\end{array}$ & $\chi^{2}$ value & $\mathrm{P}$-value & + & Positive & $\begin{array}{l}\chi^{2} \text { value } \\
\text { rate }(\%)\end{array}$ & P-value \\
\hline \multicolumn{10}{|l|}{ Age (years) } \\
\hline$<49$ & 68 & 40 & 58.8 & 0.435 & 0.510 & 25 & 36.8 & 0.997 & 0.318 \\
\hline$\geq 49$ & 64 & 34 & 53.1 & & & 29 & 45.3 & & \\
\hline \multicolumn{10}{|c|}{ Tumor size $(\mathrm{cm})$} \\
\hline$<3$ & 56 & 34 & 60.7 & 0.855 & 0.355 & 19 & 33.9 & 1.961 & 0.161 \\
\hline$\geq 3$ & 76 & 40 & 52.6 & & & 35 & 46.1 & & \\
\hline \multicolumn{10}{|l|}{ Grade } \\
\hline $\mathrm{I}+\mathrm{II}$ & 74 & 48 & 64.9 & 5.300 & $0.021^{\mathrm{a}}$ & 22 & 29.7 & 8.707 & $0.003^{\mathrm{a}}$ \\
\hline III+IV & 58 & 26 & 44.8 & & & 32 & 55.2 & & \\
\hline \multicolumn{10}{|l|}{ Stage } \\
\hline $\mathrm{I}+\mathrm{II}$ & 78 & 51 & 65.4 & 6.729 & $0.009^{\mathrm{a}}$ & 24 & 30.8 & 8.110 & $0.004^{\mathrm{a}}$ \\
\hline III+IV & 54 & 23 & 42.6 & & & 30 & 55.6 & & \\
\hline \multicolumn{10}{|c|}{ Lymphatic metastasis } \\
\hline- & 69 & 45 & 65.2 & 4.921 & $0.027^{\mathrm{a}}$ & 21 & 30.4 & 6.561 & $0.010^{\mathrm{a}}$ \\
\hline+ & 63 & 29 & 46.0 & & & 33 & 52.4 & & \\
\hline
\end{tabular}

(1), Comparison between Grade I + II and III. (2), Comparison between Stage I + II and III + IV. ${ }^{\mathrm{a} P}<0.05$.

Table III. The correlation between CacyBP and COX-2 expression in 132 cases of invasive ductal carcinoma.

\begin{tabular}{lllll}
\hline & \multicolumn{5}{c}{ COX-2 } \\
\cline { 2 - 5 } & - & + & Correlation coefficient (rs) & P-value \\
\hline CacyBP & & & 0.505 & $<0.001$ \\
- & 18 & 40 & & \\
+ & 60 & 14 & & \\
\hline
\end{tabular}

using the MTT assay. Briefly, $20 \mu \mathrm{l}$ of $5 \mathrm{mg} / \mathrm{ml} \mathrm{MTT}$ (Thiazolyl blue) solution was added to each well and incubated for $4 \mathrm{~h}$ at $37^{\circ} \mathrm{C}$. Then the media was removed from each well, and the resultant MTT formazan was solubilized in $150 \mu 1$ of DMSO. The results were quantitated spectrophotometrically at a wavelength of $490 \mathrm{~nm}$.

Statistical analysis. SPSS version 13.0 for Windows was used for all analyses. The Pearson $\chi^{2}$ test was used to examine the relationship of CacyBP or COX-2 with clinicopathological factors. The association between CacyBP and COX-2 

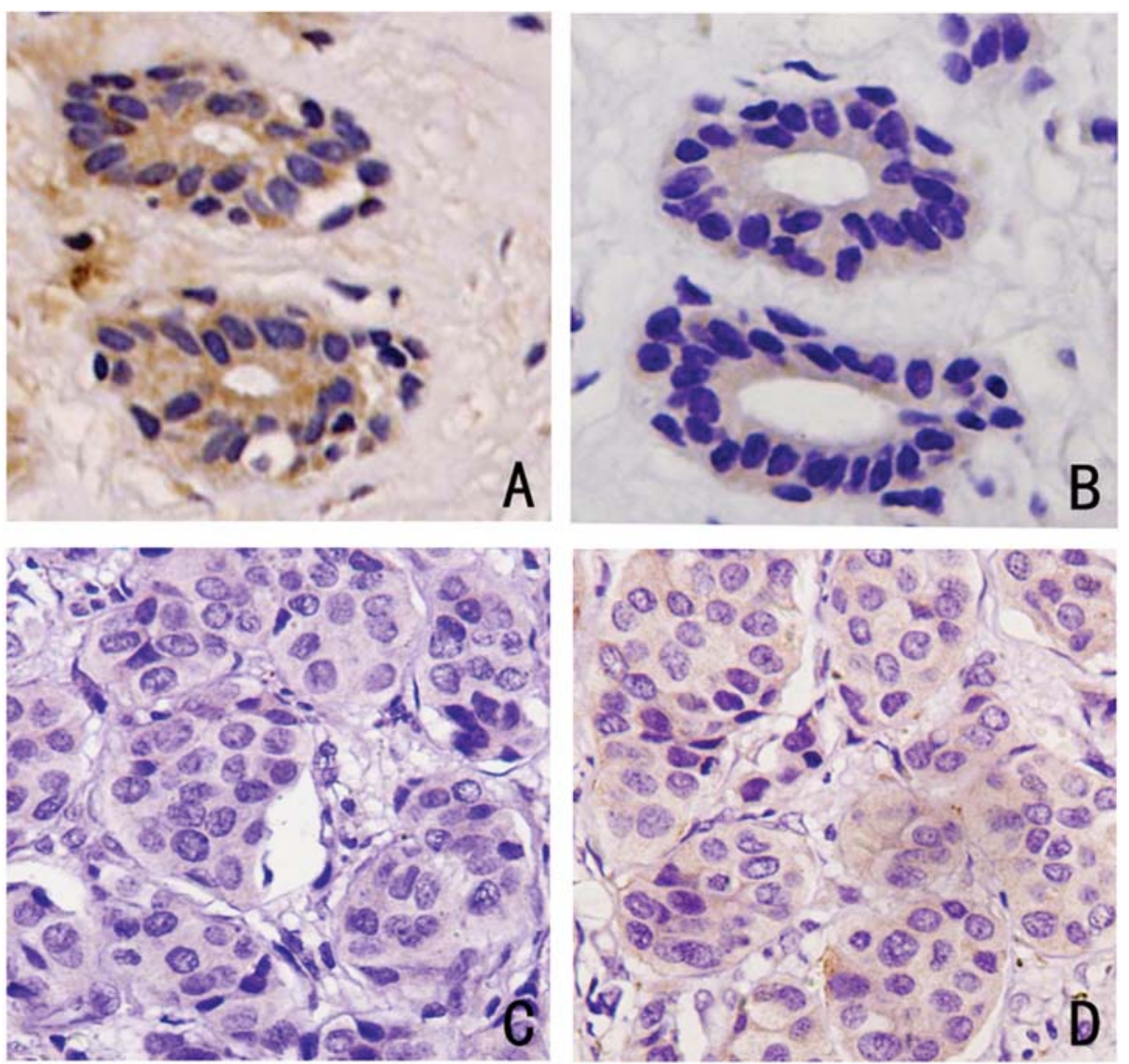

Figure 1. Breast tissues show the opposite expression of CacyBP and COX-2. The same visual field sections show CacyBP and COX-2 immunostaining in the NBT (A and B) and IDC (C and D). Original magnification $\mathrm{x} 400$.

A

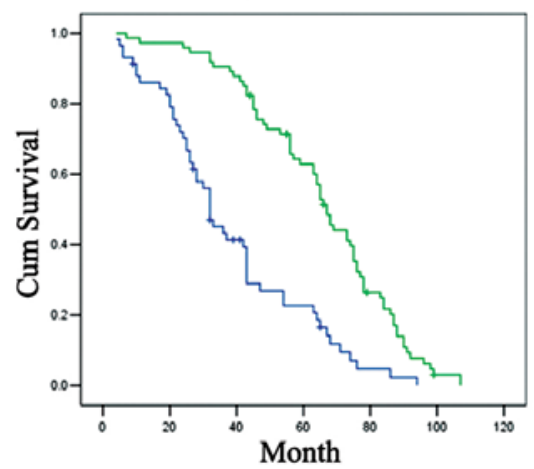

B

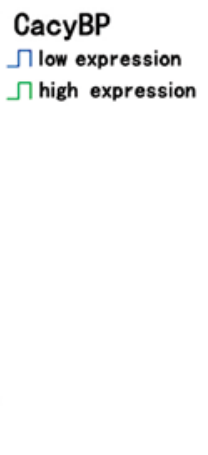

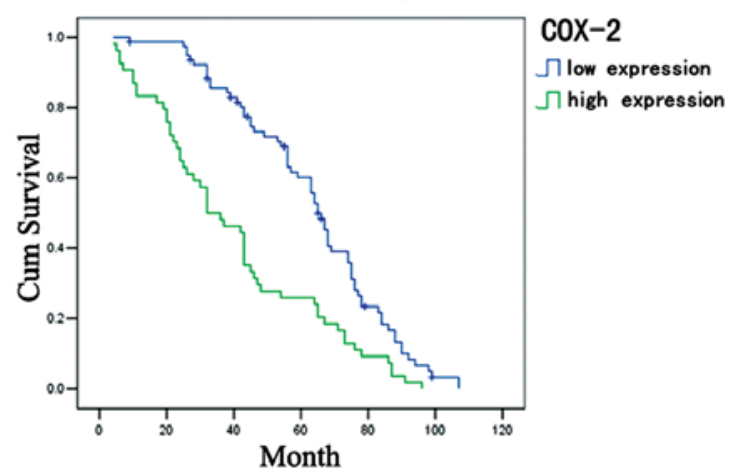

Figure 2. CacyBP and COX-2 status correlated with overall survival of breast cancer patients.

expression was assessed using the Spearman's correlation test. The Student's t-test was used to compare data from the densitometry analysis of Western blotting and RT-PCR. The Kaplan-Meier method was used to estimate the probability of patient survival.

\section{Results}

The clinical significance of the CacyBP expression in the process of breast carcinogenesis. IHC results showed that the CacyBP expression intensity in normal breast tissues (NBT)
(Fig. 1A) was stronger than in invasive ductal carcinoma (IDC) (Fig. 1C). Statistical analysis showed that the positive rate of CacyBP protein expression in NBT $(89.3 \%)$ was higher than that in IDC $(56.1 \%)(\mathrm{P}<0.05)$. The clinicopathological data analysis in 132 breast cancer samples showed that CacyBP expression was positively correlated with well differentiated samples, low pathologic TNM stage, and no lymphatic metastasis of breast cancer (Table II). The overall survival was significantly higher in patients with high expression of CacyBP breast cancer than in patients with low expression of CacyBP breast cancer ( $\mathrm{P}<0.05$, Fig. $2 \mathrm{~A})$. 


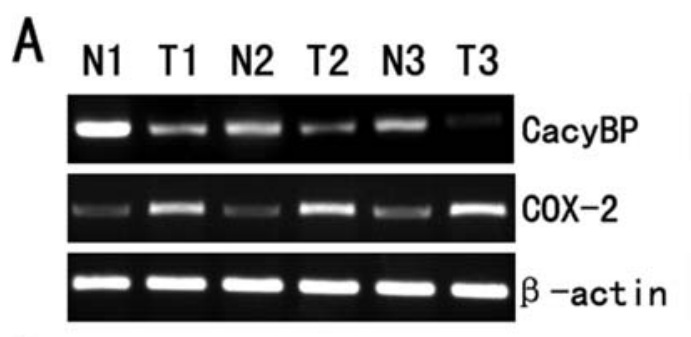

B

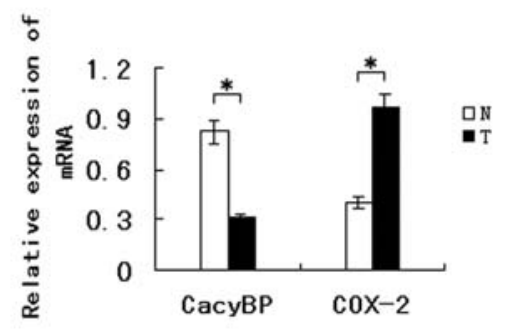

C

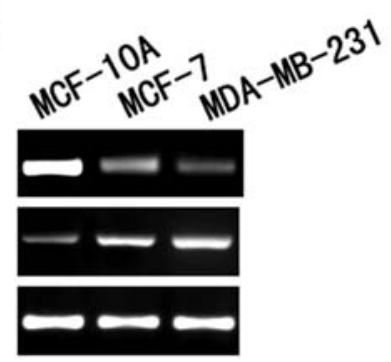

D

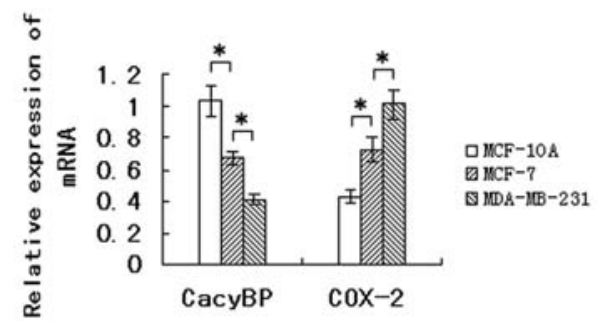

$\begin{array}{llllll}\text { N1 } & \text { T1 } & \text { N2 } & \text { T2 } & \text { N3 } & \text { T3 }\end{array}$
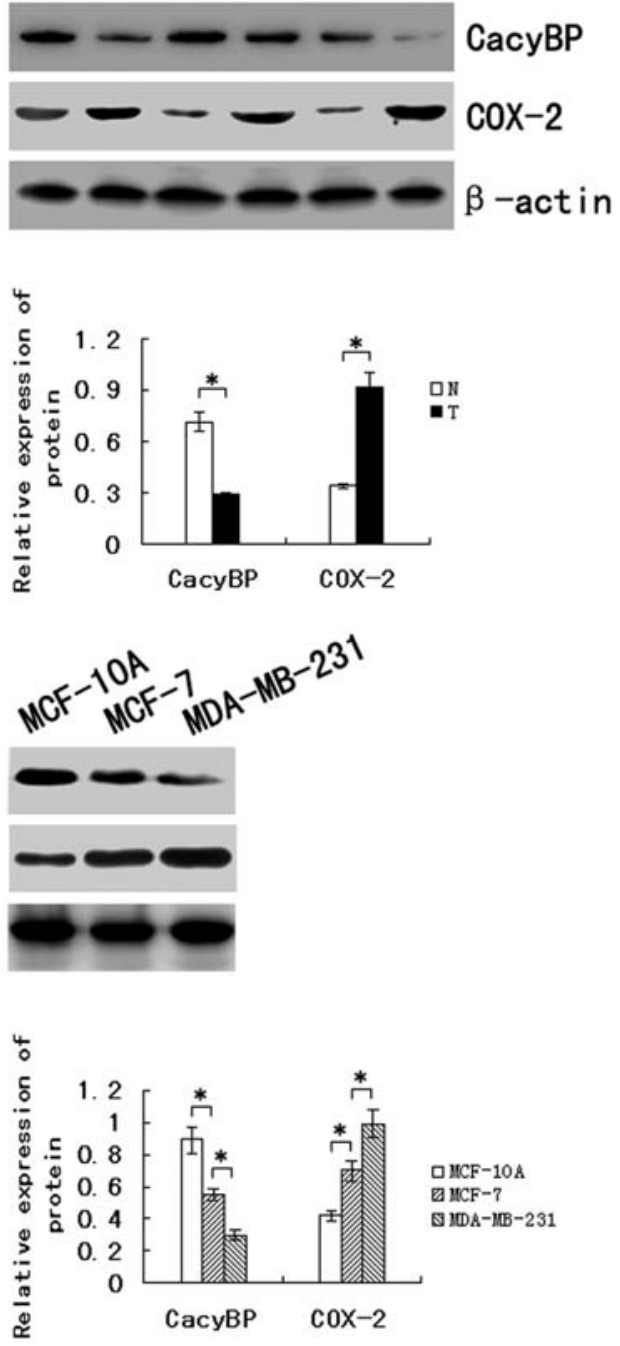

Figure 3. The expression of CacyBP and COX-2 in breast tissues and cell lines. (A) RT-PCR and Western blot analysis of CacyBP and COX-2 mRNA and protein in primary breast cancers and their adjacent normal tissues. (B) The difference of CacyBP and COX-2 expression between $\mathrm{T}$ and $\mathrm{N}$ tissues was statistically significant $(\mathrm{P}<0.05)$. ${ }^{*} \mathrm{P}<0.05$. (C) RT-PCR and Western blotting showed CacyBP and COX-2 mRNA and protein expression in human breast cell lines. (D) The difference of CacyBP and COX-2 expression in breast cell lines was statistically significant $(\mathrm{P}<0.05)$. Data represent the mean $\pm \mathrm{SD}$ of three independent experiments. Columns, mean ( $\mathrm{n}=3)$; bar, $\mathrm{SD} ;{ }^{*} \mathrm{P}<0.05$.

Western blotting and RT-PCR analysis in tissues showed that CacyBP protein $(\mathrm{P}<0.05)$ and mRNA $(\mathrm{P}<0.05)$ expression in breast cancer tissues were significantly lower than those in the corresponding non-tumorous breast tissues (Fig. 3A and B). To further detect CacyBP expression in breast cancer cell lines, we chose the MCF-7 (with low metastatic potential) and the MDA-MB-231 breast cancer cell lines (with high metastatic potential). Normal breast epithelial cell line MCF-10A was used as a control. We found that the level of CacyBP protein $(\mathrm{P}<0.05)$ and mRNA $(\mathrm{P}<0.05)$ expression in MCF-7 and MDA-MB-231 were lower than those in MCF-10A. CacyBP expression in MDA-MB-231 was lower than that in MCF-7 $(\mathrm{P}<0.05)$, which implied that CacyBP may be correlated with invasiveness and metastasis of breast cancer cells (Fig. 3C and D).

CacyBP expression was significantly negatively associated with $\mathrm{COX}-2$ expression. IHC showed that $\mathrm{COX}-2$ was located in the cytoplasm and statistical analysis showed that the positive rate of COX-2 protein expression in NBT (9.8\%) was lower than that in IDC $(40.9 \%)(\mathrm{P}<0.05)$. The representative immunohistochemical staining patterns of CacyBP (Fig. 1A and C) and COX-2 (Fig. 1B and D) compared from consecutive sections were found to show contrasting results. The Spearman's correlation test revealed that the CacyBP expression was significantly negatively associated with the COX-2 expression in the 132 breast samples $(\mathrm{P}<0.001$, Correlation coefficient $=0.505$, Table III). Statistical analysis showed that COX-2 expression was positively correlated with poor differentiation, high pathologic TNM stage, and lymphatic metastasis of breast cancer (Table II). The overall survival was significantly shorter in patients with high expression of COX-2 breast cancer than in patients with low expression of CacyBP breast cancer ( $\mathrm{P}<0.05$, Fig. 2B).

Western blotting and RT-PCR results showed COX-2 mRNA $(\mathrm{P}<0.05)$ and protein $(\mathrm{P}<0.05)$ expression were 


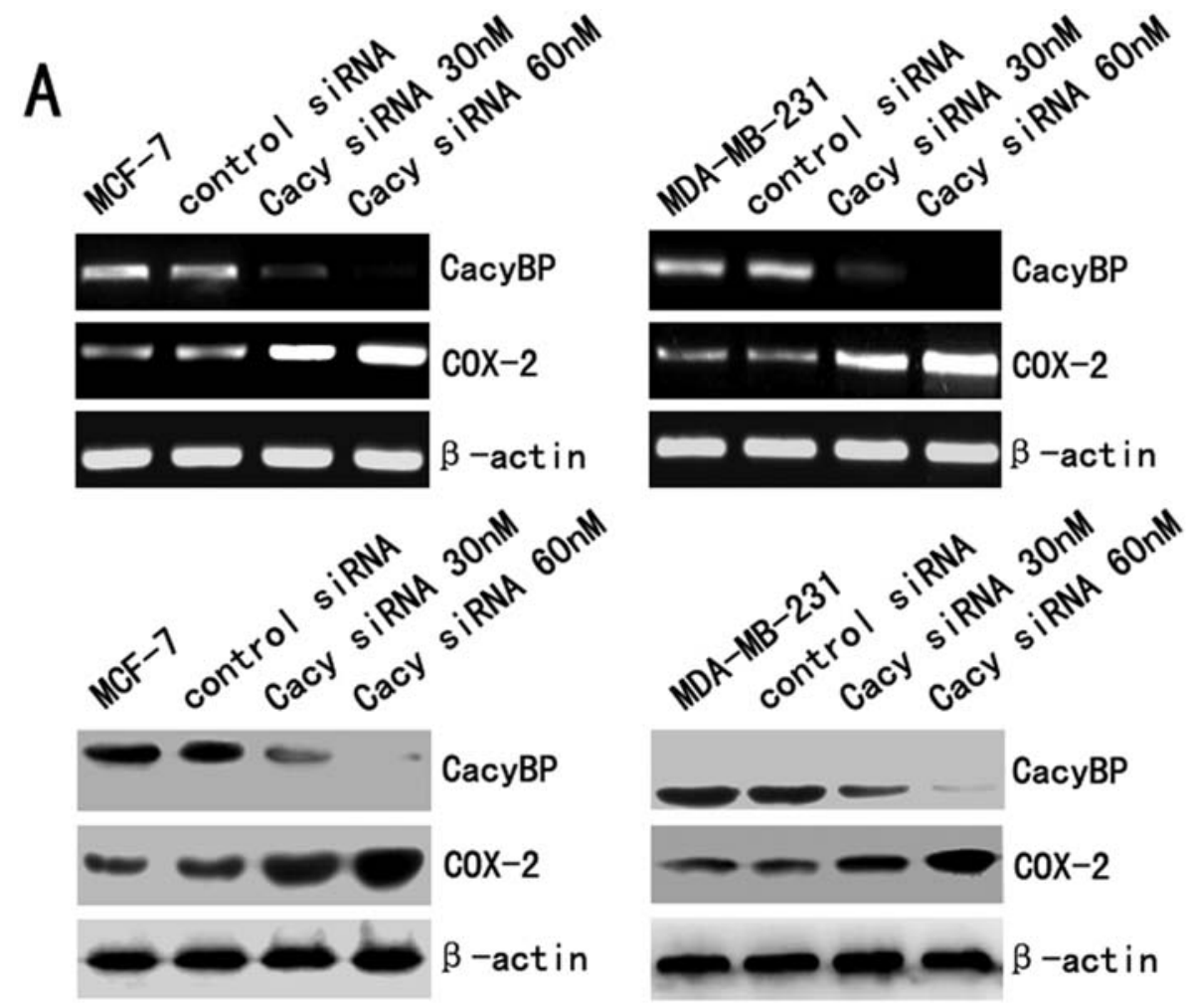

B
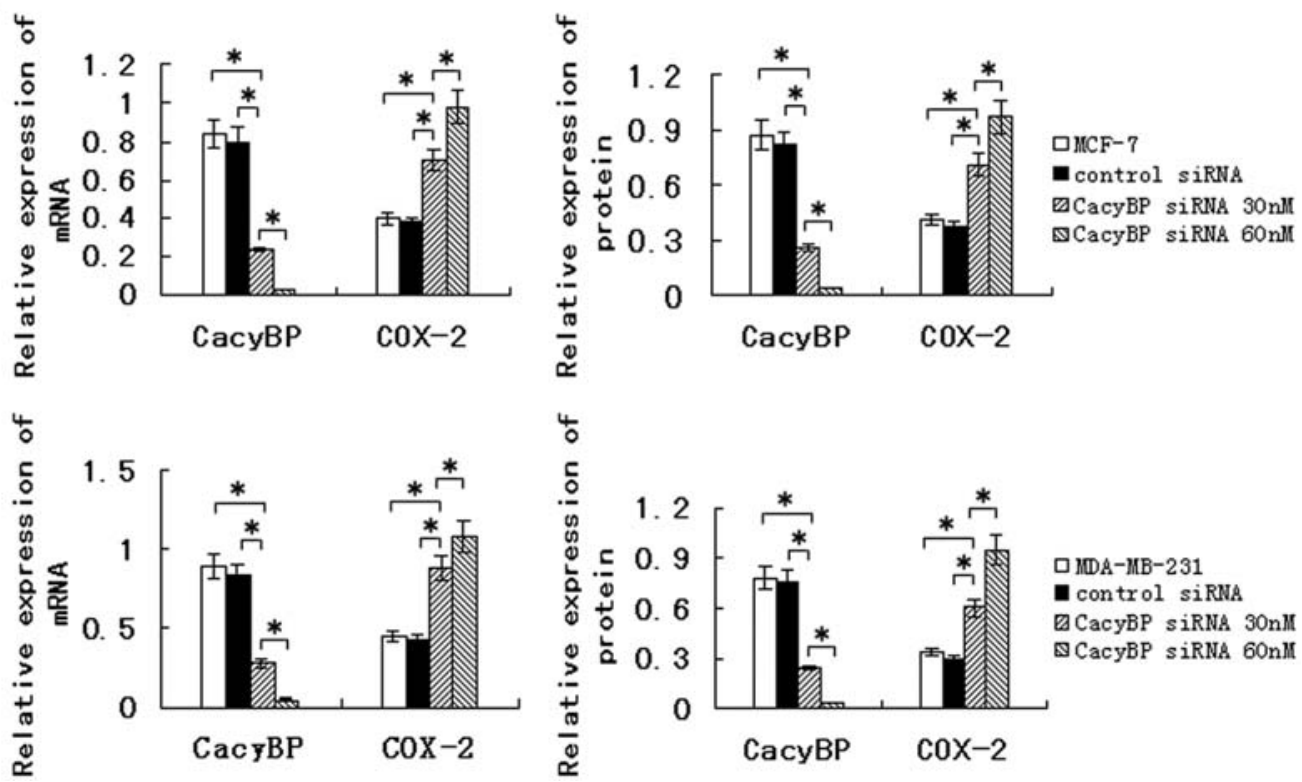

Figure 4. The knockdown of CacyBP in MCF-7 and MDA-MB-231 cell lines up-regulates COX-2 expression. (A) RT-PCR and Western blotting results showed CacyBP mRNA and protein expression were gradually decreased along with increasing concentrations of CacyBP siRNA, and COX-2 expression was gradually up-regulated along with increasing concentrations of CacyBP siRNA. (B) The difference of CacyBP and COX-2 in each division was statistically significant. Data represent the mean $\pm \mathrm{SD}$ of three independent experiments. Columns, mean ( $\mathrm{n}=3$ ); bar, $\mathrm{SD} ;{ }^{*} \mathrm{P}<0.05$.

significantly higher in 26 and 24 samples of the 30 IDC samples in comparison with the non-tumorous counterparts, respectively (Fig. 3A and B); and in human breast cancer cell lines MDA-MB-231, and MCF-7 in comparison with normal breast epithelial cell line MCF-10A (Fig. 3C and D).

The knockdown of CacyBP up-regulates $C O X-2$ expression. To further explore the relationship between CacyBP and
COX-2, we employed CacyBP siRNA to knock down CacyBP expression in MCF-7 and MDA-MB-231 cells. RT-PCR and Western blotting were used to examine the effectiveness of siRNA. CacyBP gradually decreased along with increasing CacyBP siRNA concentration, and CacyBP expression was barely detectable at the concentration of $60 \mathrm{nM}$. Increasing CacyBP siRNA concentration led to a significant upregulation of COX-2 expression (Fig. 4). 


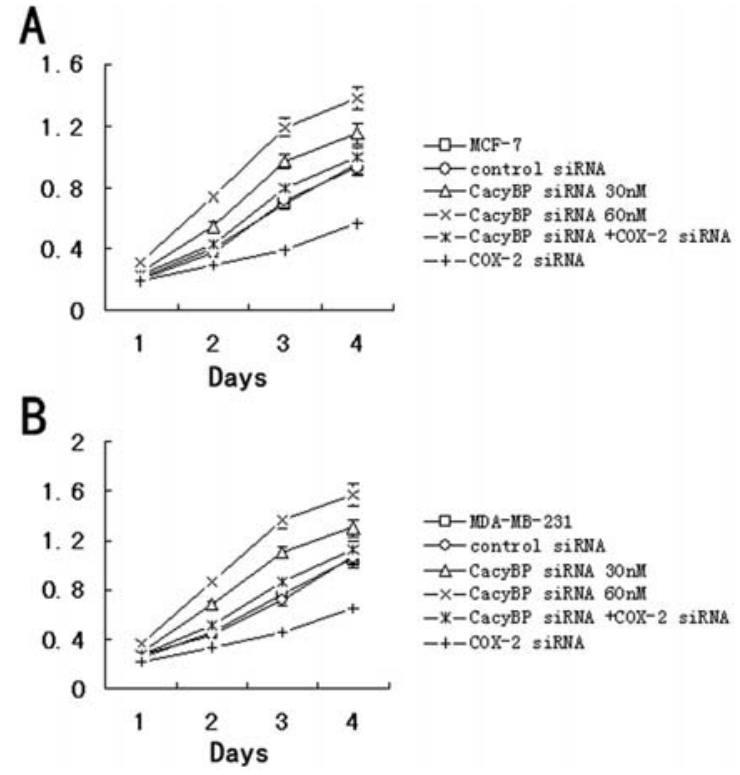

Figure 5. The knockdown of CacyBP increases cancer cell proliferation dependent on COX-2. MTT assay was performed after CacyBP siRNA treatment. The level of proliferation was significantly lower in cells cotransfected with CacyBP siRNA and COX-2 siRNA than in cells transfected with CacyBP siRNA alone. Data represent the mean \pm SD of three independent experiments. Columns, mean ( $\mathrm{n}=3)$; bar, $\mathrm{SD} ;{ }^{*} \mathrm{P}<0.05$.
The knockdown of CacyBP promotes breast cancer cell growth dependent on COX-2. To determine the influence of CacyBP on the proliferation of breast cancer cells, we performed MTT to examine the change in the proliferation ability. A significant increase was observed in the proliferation rate of MCF-7 and MDA-MB-231 cells transfected with CacyBP siRNA compared with the proliferation rate of the cells transfected with negative control siRNA [ $\mathrm{P}>0.05$ (day 1); $\mathrm{P}<0.01$ (days 2-4), $\mathrm{n}=3]$. The cells co-transfected with CacyBP siRNA and COX-2 siRNA had lower proliferation rate than cells transfected with CacyBP siRNA [P>0.05 (day 1); $\mathrm{P}<0.01$ (days 2-4), $\mathrm{n}=3$ ] (Fig. 5).

The knockdown of CacyBP increases the invasive ability of breast cancer cells dependent on $C O X-2$. Matrigel invasion assays showed that the invasive ability of the MCF-7 and MDA-MB-231 cells gradually enhanced along with increasing concentrations of CacyBP siRNA $(\mathrm{P}<0.05)$. MCF-7 and MDA-MB-231 cells co-transfected with CacyBP siRNA and COX-2 siRNA had fewer invasive cells than that transfected with CacyBP siRNA alone $(\mathrm{P}<0.05)$ (Fig. 6).
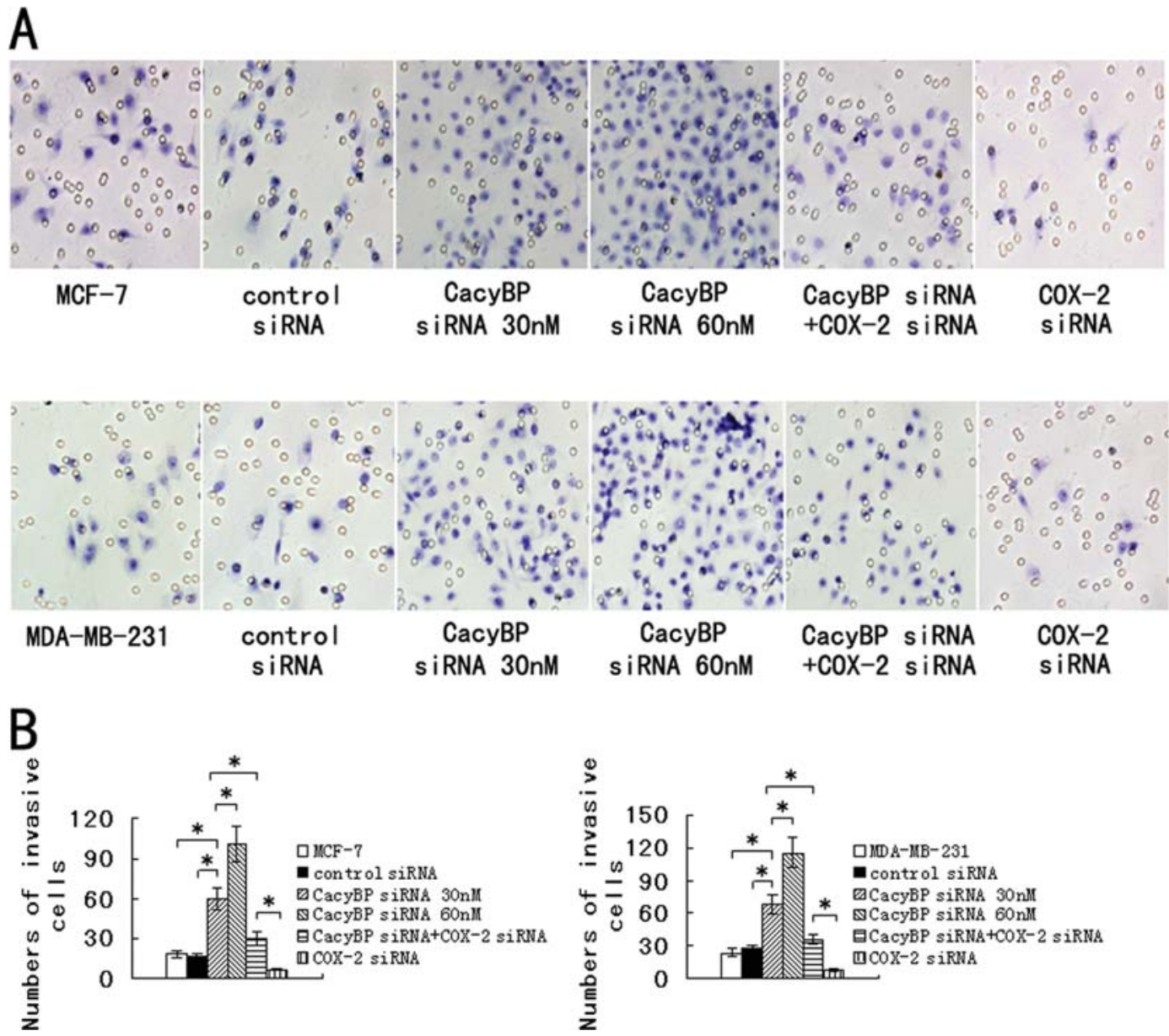

Figure 6. The knockdown of CacyBP increases the invasive ability of breast cancer cells dependent on COX-2. (A) Matrigel invasion assay showed that the invasive cells gradually increased along with increasing doses of CacyBP siRNA in cells. The cells co-transfected with CacyBP siRNA and COX-2 siRNA had fewer cells invading onto the lower surface of the Transwell filter than the cells transfected with CacyBP siRNA alone. (B) Number of cells invading onto the lower surface of the filter was counted. Data represent the mean $\pm \mathrm{SD}$ of three independent experiments. Columns, mean $(\mathrm{n}=3)$; bar, $\mathrm{SD}$; ${ }^{\mathrm{P}<0.05 \text {. }}$ 


\section{Discussion}

CacyBP functions as a tumor suppressor in gastric cancer and renal cell carcinoma (9), but as an oncogene in pancreatic cancer (10). However, the function of CacyBP in breast cancer has not been previously elucidated. In the present study, we demonstrated that the expression of CacyBP was significantly decreased in breast carcinogenesis. The level of CacyBP in breast cancer tissues was significant lower than that in normal breast tissues at both protein and mRNA levels. Importantly, reduced CacyBP expression was associated with poor prognosis of breast cancer patients. Furthermore, CacyBP expression was significantly negatively associated with COX-2 expression. In addition, we found that depletion of CacyBP in breast cancer cells led to increase cell proliferation and invasion by up-regulating COX-2 expression.

Previous studies showed that CacyBP mRNA and protein expression were higher in a well-differentiated gastric cancer cell line than those in poorly differentiated gastric cancer cell line and its expression was associated with the malignant phenotype of gastric cancer cells (8). Studies in renal cancer demonstrated that CacyBP expression was reduced in cancerous tissues and cells (9). These observations suggested that CacyBP might be a tumor suppressor in many human malignancies. In accordance with these findings, our report showed there was a down-regulation of CacyBP expression in breast cancer. Moreover, both mRNA and protein levels of CacyBP were lower in tumor than those in paired non-tumorous counterparts. Importantly, reduced CacyBP expression was significantly correlated with poorly differentiated tumors, higher TNM stage, lymphatic metastasis, and reduced overall survival for breast cancer patients. These results suggested that the loss or reduced expression of CacyBP played an important role in breast carcinogenesis. In contrast to these studies, some studies reported that CacyBP expression was higher in pancreatic cancer compared to adjacent tissues and correlated with poorly differentiation, and higher TNM stage (10). Therefore, CacyBP may have different influence on cancer cells in different cancer types.

It has been shown that overexpressed CacyBP could inhibit cell proliferation and invasion in gastric cancer (7) and renal cell carcinoma (9). However, the effect of CacyBP on breast cancer cells is unclear. In our study, we found that depletion of CacyBP promoted breast cancer cell proliferation and invasion. The mechanisms responsible for promotion of cell proliferation and invasion by knockdown of CacyBP still need to be clarified. Some studies indicated that CacyBP might modulate the malignant behavior by regulating the level of $\beta$-catenin (7). CacyBP participated in the p53induced $\beta$-catenin degradation (12). B-catenin functioned as an oncogene in many different types of cancers $(13,14)$. It has been reported that $\beta$-catenin could up-regulate COX-2 expression in human cancer cells $(15,16)$, and $\beta$-catenin involved in the activation of the COX-2 promoter by wnt signaling pathway (17). COX-2 was overexpression in colorectal, gastric, lung and breast carcinomas, and it was involved in the processes of carcinogenesis, cell survival, invasion, metastasis and poor clinical outcome (18-22).
Interestingly, we found that CacyBP was significantly negatively correlated with the expression of COX-2 in breast tissues, indicating that CacyBP might have some direct or indirect effects on COX-2 expression. So we hypothesized that CacyBP might regulate proliferation and invasion of breast cancer cells in part by modulating COX-2 expression. Then we introduced CacyBP siRNA into the breast cancer cells, and found that the depletion of CacyBP expression upregulated the level of COX-2. To further investigate the role of COX-2 in CacyBP-inhibition proliferation and invasion of breast cancer cells, cells were co-transfected with CacyBP siRNA and COX-2 siRNA, and we found that knockdown of CacyBP-induced proliferation and invasion of breast cancer cells was reversed, which also confirmed that COX-2 took part in the knockdown of CacyBP-induced proliferation and invasion of breast cancer cells.

In conclusion, this study identified $C a c y B P$ as an antitumor gene in breast carcinogenesis. CacyBP plays an important role in inhibiting cell growth, indicating $C a c y B P$ gene is a potential therapeutic target of breast cancer.

\section{References}

1. Debatin K: Activation of apoptosis pathways by anticancer treatment. Toxicol Lett 112-113: 41-48, 2000.

2. Shao ZM, Li J, Wu J, Han QX, Shen ZZ, Fontana JA and Barsky SH: Neo-adjuvant chemotherapy for operable breast cancer induces apoptosis. Breast Cancer Res Treat 53: 263-269, 1999.

3. Filipek A and Wojda U: p30, a novel protein target of mouse calcyclin (S100A6). Biochem J 320: 585-587, 1996.

4. Filipek A and Kuznicki J: Molecular cloning and expression of a mouse brain cDNA encoding a novel protein target of calcyclin. J Neurochem 70: 1793-1798, 1998.

5. Filipek A, Jastrzebska B, Nowotny M and Kuznicki J: CacyBP/ SIP, a calcyclin and Siah-1-interacting protein, binds EF-hand proteins of the S100 family. J Biol Chem 277: 28848-28852, 2002.

6. Zhai H, Shi Y, Jin H, Li Y, Chen X, Wang J, Ding L, Wang X and Fan D: Expression of Calcyclin-binding protein/siah-1 interacting protein in normal and malignant human tissues: an immunohistochemical survey. J Histochem Cytochem 56: 765-772, 2008.

7. Ning X, Sun S, Hong L, Liang J, Liu L, Han S, Liu Z, Shi Y, Li Y, Gong W, Zhang S, Chen Y, Guo X, Cheng Y, Wu K and Fan D: Calcyclin-binding protein inhibits proliferation, tumorigenicity, and invasion of gastric cancer. Mol Cancer Res 5: 1254-1262, 2007.

8. Yang YJ, Liu WM, Zhou JX, Cao YJ, Li J, Peng S, Wang L, Yuan JG and Duan EK: Expression and hormonal regulation of calcyclin-binding protein (CacyBP) in the mouse uterus during early pregnancy. Life Sci 78: 753-760, 2006.

9. Sun S, Ning X, Liu J, Liu L, Chen Y, Han S, Zhang Y, Liang J, Wu K and Fan D: Overexpressed CacyBP/SIP leads to the suppression of growth in renal cell carcinoma. Biochem Biophys Res Commun 356: 864-871, 2007.

10. Chen X, Han G, Zhai H, Zhang F, Wang J, Li X, Huang S, Wang $X$ and Fan D: Expression and clinical significance of CacyBP/SIP in pancreatic cancer. Pancreatology 8: 470-477, 2008.

11. Ristimaki A, Sivula A, Lundin J, Lundin M, Salminen T, Haglund $\mathrm{C}$, Joensuu $\mathrm{H}$ and Isola J: Prognostic significance of elevated cyclooxygenase- 2 expression in breast cancer. Cancer Res 62: 632-635, 2002.

12. Matsuzawa SI and Reed JC: Siah-1, SIP, and Ebi collaborate in a novel pathway for beta-catenin degradation linked to p53 responses. Mol Cell 7: 915-926, 2001.

13. Fuchs SY, Ougolkov AV, Spiegelman VS and Minamoto T: Oncogenic beta-catenin signaling networks in colorectal cancer. Cell Cycle 4: 1522-1539, 2005.

14. Peifer M and Polakis P: Wnt signaling in oncogenesis and embryogenesis - a look outside the nucleus. Science 287 : 1606-1609, 2000. 
15. Araki Y, Okamura S, Hussain SP, Nagashima M, He P, Shiseki M, Miura K and Harris CC: Regulation of cyclooxygenase- 2 expression by the wnt and ras pathways. Cancer Res 63: 728-734, 2003

16. Wang H, Wen S, Bunnett NW, Leduc R, Hollenberg MD and Macnaughton WK: Proteinase-activated receptor-2 induces cyclooxygenase-2 expression through beta-catenin and cyclic AMP-response element-binding protein. J Biol Chem 283: 809-815, 2008

17. Krysan K, Reckamp KL, Sharma S and Dubinett SM: The potential and rationale for COX-2 inhibitors in lung cancer. Anticancer Agents Med Chem 6: 209-220, 2006.

18. Chow LW, Loo WT and Toi M: Current directions for COX-2 inhibition in breast cancer. Biomed Pharmacother 59: S281-S284, 2005.
19. Amir M and Agarwal HK: Role of COX-2 selective inhibitors for prevention and treatment of cancer. Pharmazie 60: 563-570, 2005.

20. Brown JR and Dubois RN: COX-2: a molecular target for colorectal cancer prevention. J Clin Oncol 23: 2840-2855, 2005.

21. Blanke C: Role of COX-2 inhibitors in cancer therapy. Cancer Invest 22: 271-282, 2004

22. Sheehan KM, Sheahan K, O'Donoghue DP, MacSweeney F, Conroy RM, Fitzgerald DJ and Murray FE: The relationship between cyclooxygenase- 2 expression and colorectal cancer. JAMA 282: 1254-1277, 1999. 